\title{
The effect of an electric field on the thermomechanical damage of nodular defects in dielectric multilayer coatings irradiated by nanosecond laser pulses
}

\author{
Xinbin Cheng ${ }^{1,2}$, Jinlong Zhang ${ }^{1,2}$, Tao Ding ${ }^{1,2}$, Zeyong Wei ${ }^{1}$, Hongqiang $\mathrm{Li}^{1}$ and Zhanshan Wang ${ }^{1,2}$
}

Thermomechanical damage of nodules in dielectric multilayer coatings that are irradiated by nanosecond laser pulses has been interpreted with respect to mechanical properties and electric-field enhancement. However, the effect of electric-field enhancement in nodular damage, especially the influence of electric-field distributions, has never been directly demonstrated through experimental results, which prevents the achievement of a clear understanding of the damage process of nodular defects. Here, a systematic and comparative study was designed to reveal how electric-field distributions affect the damage behavior of nodules. To obtain reliable results, two series of artificial nodules with different geometries and film absorption characteristics were prepared from monodisperse silica microspheres. After establishing simplified geometrical models of the nodules, the electric-field enhancement was simulated using a three-dimensional finite-difference time-domain code. Then, the damage morphologies of the artificial nodules were directly compared with the simulated electric-field intensity profiles. For both series of nodules, the damage morphologies reproduced our simulated electric-field intensity distributions very well. These results indicated that the electric-field distribution was actually a bridge that connected the nodular mechanical properties to the final thermomechanical damage. Understanding of the damage mechanism of nodules was deepened by obtaining data on the influence of electric-field distributions on the damage behavior of nodules.

Light: Science \& Applications (2013) 2, e80; doi:10.1038/Isa.2013.36; published online 21 June 2013

Keywords: electric field; nanosecond-pulse-laser damage; nodular defects; thin films

\section{INTRODUCTION}

The performance of optical components facilitates technologies for high-power laser systems. Their performance, especially with regard to laser damage resistance, has a significant influence on the design, construction cost, safe operation and service life of fusion-level nanosecond laser systems such as the National Ignition Facility, ${ }^{1}$ Laser Megajoule, ${ }^{2}$ Shenguang, ${ }^{3}$ etc. Numerous experimental and theoretical studies have been carried out to understand the damage mechanisms of optical components. ${ }^{4-9}$ As our understanding of these damage mechanisms deepens, existing technologies can be re-optimized, or new processes can be developed to increase the laser-induced damage threshold (LIDT) beyond any level previously attained.

Near-infrared high-reflection (HR) coatings and polarizers are widely used in nanosecond high-power laser systems. The damage mechanisms of these dielectric multilayers have been studied extensively to achieve the maximum LIDTs possible. Nodules were identified as the predominant factors that decrease the LIDT; these nodules are defects that grow from seeds or particulates into an inverted conical shape with a domed top protruding above the surface of the film. ${ }^{10}$ A simple model has been proposed to represent the geometries of classic nodules, where the nodular diameter $D$ is related to a constant
$C$, to the seed diameter $d$, and to the seed depth $t$ via the relation $D=\operatorname{sqrt}(C d t) .{ }^{11}$ For a rotating substrate in an electron beam evaporation (EBE) process, nodular growth exhibits a self-shadowing nature, and the constant $C$ is dependent on deposition conditions such as the coater geometry and the mobility of deposited atoms. A simulation of nodular growth by Liao et al. ${ }^{12}$ showed that a greater nodular diameter would result from either a larger incident angle of evaporated atoms or a lower mobility of atoms.

The mechanical stability of nodules is poor because the boundaries between the nodules and thin films are usually discontinuous, with a great many voids being present. When laser irradiation induces a strong temperature gradient and associated stress, thermomechanical damage initiates preferentially at the unstable nodular boundaries. Models based on the geometrical enhancement of the electric-field intensity $\left(|E|^{2}\right)$ have been proposed to understand nodular ejection mechanisms. DeFord et al. made the first finite-difference timedomain (FDTD) model of $|E|^{2}$ enhancement in nodular defects and demonstrated that a nodule could result in an electric-field enhancement that was 3.5 times higher than the incident field strength. ${ }^{13}$ However, their code limited calculations to two-dimensional cases only and also neglected polarization effects. In fact, a nodule is exposed

${ }^{1}$ Key Laboratory of Advanced Micro-Structure Materials, Ministry of Education, Shanghai 200092, China and ${ }^{2}$ Institute of Precision Optical Engineering, School of Physics Science and Engineering, Tongji University, Shanghai 200092, China

Correspondence: Professor ZS Wang, Institute of Precision Optical Engineering, School of Physics Science and Engineering, Tongji University, Shanghai 200092, China

E-mail: wangzs@tongji.edu.cn

Received 28 January 2013; revised 1 March 2013; accepted 5 March 2013 
to a range of incident angles and to opposite polarizations at orthogonal cross-sections, so only a three-dimensional (3D) FDTD code can sufficiently mimic the $|E|^{2}$ enhancement in nodules.

The first $|E|^{2}$ simulation of nodules using a 3D FDTD code was accomplished by Stolz et al. ${ }^{14}$ They subsequently carried out in-depth studies to investigate the dependence of $|E|^{2}$ enhancement on the seed diameter, lodging depth, incident angle, centering wavelength, etc. ${ }^{15}$ Their modeling results showed that light intensification could occur in nodules by a factor as large as 24 . Since then, it has been well accepted that $|E|^{2}$ enhancement is an important cause of nodular ejection, and the measured LIDT of nodules has been related to the peak $|E|^{2}$ of the nodules. ${ }^{16}$ However, $|E|^{2}$ simulations of nodules are far from comprehensive. Nodules with different aspect ratios have been reported, ${ }^{17,18}$ but systematic studies of electric-field modeling of nodules were limited to a $D=\operatorname{sqrt}(8 d t)$ geometry. The influence of nodular geometries on $|E|^{2}$ enhancement is still not clear.

Although it is now well known that $|E|^{2}$ triggers laser damage in nodules, understanding of how $|E|^{2}$ distributions affect the damage behavior of nodules is still vague. Indeed, the $|E|^{2}$ distribution determines the spatial distribution of the absorbed laser energy in the area surrounding a nodule, knowledge of which is an important precondition to understanding the subsequent complex processes of temperature distribution, stress response and mechanical damage. ${ }^{19}$ Without clear knowledge of the $|E|^{2}$ distributions and of how those distributions affect the damage behavior of nodules, it is impossible to gain a true understanding of the thermomechanical damage process of nodules. The reason why previous studies failed to find a dependence of nodular damage on $|E|^{2}$ distributions is easy to explain. In practice, the density of nodules is usually very low, and their properties are quite diverse. It is very time-consuming and quite challenging to obtain representative damage morphologies of nodules and to make meaningful comparisons to simulation results. Artificial nodules whose density, size, absorption and seed lodging depth can be well controlled have been used to study damage behaviors of nodules in a more reliable and efficient way. ${ }^{17,18,20}$ Unfortunately, the studies that used artificial nodules either did not consider the damage mechanism with respect to $|E|^{2}$ distributions or used a two-dimensional FDTD code that could not adequately mimic the $|E|^{2}$ distributions in nodules.

In this work, an experimental and theoretical study was designed to investigate the influence of $|E|^{2}$ distributions on the damage behavior of nodules. The reliability of the experimental study on artificial nodules was further improved by creating them from monodisperse silica microspheres. $|E|^{2}$ profiles on two different nodular geometries were simulated using a 3D FDTD code. In addition, the link between $|E|^{2}$ distributions and the thermomechanical damage of nodules was explored by direct comparison of simulated $|E|^{2}$ patterns and damage morphologies. Our results demonstrate exactly how $|E|^{2}$ distributions affect the damage behavior of nodular defects irradiated by nanosecond laser pulses.

\section{MATERIALS AND METHODS}

\section{Preparation of artificial nodules}

$\mathrm{SiO}_{2}$ microspheres were selected as seeds because both their properties and monodispersity could be guaranteed using well-established technology. Such a guarantee is quite important for obtaining representative damage morphologies of the resulting nodules. For practical interest, the size of $\mathrm{SiO}_{2}$ microspheres investigated ranged from hundreds of nanometers to several micrometers, and the only condition considered was that the seed be located on the substrate surface. ${ }^{18}$ Monodisperse silica microspheres having five distinct sizes-0.3,
$0.6,0.9,1.45$ and $1.9 \mu \mathrm{m}$-were prepared using the Stöber process. These prepared seeds were then deposited onto the surface of BK7 substrates in a well-dispersed manner via a spin-coating method. Both the concentration of the aqueous suspension containing the silica microspheres and the spin speed were adjusted to achieve an average areal density of $20-40 \mathrm{~mm}^{-2}$, which is optimal for laser damage testing to obtain representative damage morphologies of the artificial nodules. For each type of silica microsphere, $12 \mathrm{BK} 7$ substrates were prepared for subsequent deposition of $\mathrm{HfO}_{2} / \mathrm{SiO}_{2} \mathrm{HR}$ coatings. To better illustrate why two deposition processes were adopted, the deposition conditions for each process will be described in detail in the following sections.

\section{D FDTD algorithm}

The $|E|^{2}$ distributions were simulated using a 3D FDTD electromagnetic code. $^{21}$ The simulation domain was rectangular, 3D and non-uniformly gridded. To obtain accurate simulation results, the rectangular simulation domain was gridded with spacings that were sufficiently small to ensure that there were at least 15 samples per wavelength. Furthermore, our algorithm took interfaces as mesh nodes and ensured that the thickness of each layer was accurate after gridding. Periodic boundary conditions were applied in the $x$ - and $y$-directions, while perfectly matched layer-absorbing boundary conditions were applied in the $z$-direction. To reduce back-reflections from the periodic boundary conditions, a simulation domain $24 \mu \mathrm{m}$ wide and $7.5 \mu \mathrm{m}$ high was used for nodules initiating from the $1.9 \mu \mathrm{m}$ seeds. A simulation domain $16 \mu \mathrm{m}$ wide and $7.5 \mu \mathrm{m}$ high was used for the other nodules initiating from smaller seeds to reduce the computation time. The simulation was performed using a plane wave with a center wavelength of $1064 \mathrm{~nm}$ as the incident field.

\section{Laser damage test}

Damage studies of the artificial nodules were carried out in our laser damage test facility. The system employed a Q-switched $1064 \mathrm{~nm}$ Nd:YAG laser from Spectra-Physics (Santa Clara, CA, USA). The output laser beam has a $\mathrm{TEM}_{00}$ mode with a pulsewidth of $10 \mathrm{~ns}$ and a repetition rate of $10 \mathrm{~Hz}$. A raster scan method was used to obtain representative damage morphologies of artificial nodules. To avoid repeat irradiation on the same nodule, the beam was transported by the beam diameter at an intensity of $1 / e^{2}$ where the size was approximately $1 \mathrm{~mm}$. The raster scan was halted when either more than 100 nodules were ejected or catastrophic damage occurred. The laser energy originated from a specific fluence that was based on previous experience and that was gradually increased to the 'stop' fluence using increments of $5 \mathrm{~J} \mathrm{~cm}^{-2}$. The maximum output power on the sample surface was $170 \mathrm{~J} \mathrm{~cm}^{-2}$. Following the damage test, the damage morphologies of the ejected nodules were identified first under a Nomarski microscope and were then characterized using focused ion beam (FIB) technology.

\section{RESULTS AND DISCUSSION}

\section{Thermomechanical damage of artificial nodules prepared by the} EBE process

EBE is our primary process for depositing HR coatings because of its potential to achieve coatings possessing the highest LIDT. Thus, artificial nodules prepared via EBE were investigated first. The details of our modified EBE process using oxygen radicals were given in a previous study. ${ }^{22} \mathrm{HfO}_{2} / \mathrm{SiO}_{2} \mathrm{HR}$ coatings with a wavelength of $1.064 \mu \mathrm{m}$ and normal incidence angle were used in this study. The design was [air: $\mathrm{L}(\mathrm{LH})^{\wedge}$ glass], where $\mathrm{H}$ is a quarter-wave $\mathrm{HfO}_{2}$ layer, and $\mathrm{L}$ is a 

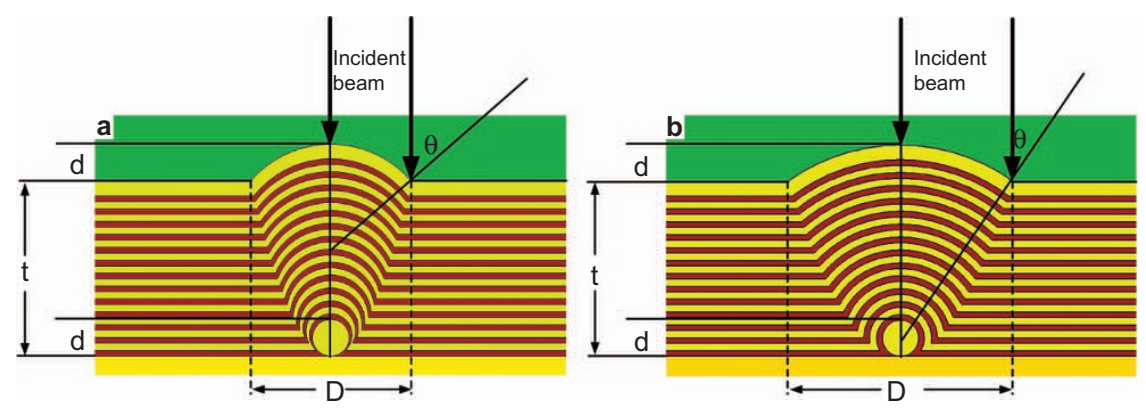

Figure 1 Nodular geometries used for electric-field simulations. (a) Nodule with a $D=$ sqrt( $4 d t$ ) aspect ratio initiating from a $0.9-\mu \mathrm{m}$ silica microsphere for which the film thickness over the seed is non-uniform. (b) Nodule with a $D=s q r t(8 d t)$ aspect ratio initiating from a $0.9-\mu$ m silica microsphere for which the film thickness over the seed is uniform. The incident angle across the nodular surface is non-constant. At the nodular center, the incident angle is zero, and it increases to a maximum angle $\theta$ when moving to the nodular edge.

quarter-wave $\mathrm{SiO}_{2}$ layer. The refractive indices of $\mathrm{HfO}_{2}$ and $\mathrm{SiO}_{2}$ are 1.962 and 1.453 , respectively, at $1.064 \mu \mathrm{m}$. The total film thickness was approximately $4.32 \mu \mathrm{m}$. The absorption of the HR coatings was measured using a photothermal common path interferometer and was 4.5 parts per million at a normal incidence of $1.064 \mu \mathrm{m}$.

\section{Geometric modeling of artificial nodules}

Cross-sectional micrographs of prepared nodules have previously been examined to reveal the nodular geometries. ${ }^{18}$ These results showed that nodules prepared via EBE possessed an aspect ratio of $D=\operatorname{sqrt}(4 d t)$. In this study, however, three additional conditions were utilized to establish the geometrical model of nodules with a $D=\operatorname{sqrt}(4 d t)$ aspect ratio for FDTD simulations. The first condition applied was that the nodular height is equal to the seed diameter. The second condition utilized was that the layers growing radially outward from the spherical seeds are spherical sections. Finally, the third condition applied was that voids along the boundaries of nodules are neglected.

Based on these additional conditions, simplified 3D geometrical models of nodules with a $D=\operatorname{sqrt}(4 d t)$ aspect ratio were established. For example, Figure 1a shows a cross-sectional geometrical model of a nodule with a $D=\operatorname{sqrt}(4 d t)$ aspect ratio initiating from a $0.9 \mu \mathrm{m}$ seed. The resulting layers that form above the seed are tangent spheres, and the point of tangency is the intersection point between the spherical seed and the substrate surface. It is worth noting that this geometrical model leads to the condition that the thickness of a film growing radially outward from a spherical seed is non-uniform and is also different from the thickness of a vertical layer film growing on a perfect substrate, except along the central axis of the nodule. The nonuniformity of the film thickness growing radially from the spherical seed is quite pronounced for the first several deposited layers and gradually becomes negligible for subsequent deposited layers.

This phenomenon is different from the case of the widely used $D=\operatorname{sqrt}(8 d t)$ geometrical model in which the resulting layers that form above the seed are concentric spheres and in which the thickness of a film growing radially outward from the spherical seeds is the same as the thickness of a vertical layer film growing on a perfect substrate. For comparison, the $D=\operatorname{sqrt}(8 d t)$ geometrical model is shown in Figure $1 \mathrm{~b}$. We think that the shadowing effects of seeds most likely result in the non-uniform thickness of deposited layers on seeds and that the deposition rates of evaporated atoms actually vary as a function of the incident angle from which they arrive. The non-uniform thickness of the layer above the spherical seed is thought to be reasonable, and to a certain degree, it reflects the real nodular structure.
However, it is quite difficult to justify this assumption by evaluating cross-sectional micrographs of the nodules due to the existence of voids along the nodular boundaries. The soundness of this nonuniform geometrical model must be proven through experimental results. Moreover, the influence of voids on the $|E|^{2}$ enhancement in nodules is not yet understood. The validity of neglecting voids in the model should also be confirmed through experimental results.

\section{Spectral characteristics of artificial nodules}

Before providing the results of the $|E|^{2}$ simulation, we will first address the influence of the geometrical model on the spectral characteristics of nodules. Although the $\mathrm{HfO}_{2} / \mathrm{SiO}_{2} \mathrm{HR}$ coatings are irradiated at a normal incidence, the nodules are exposed to a range of incident angles as well as to both S-polarization and P-polarization at orthogonal cross-sections. When the point of incidence moves from the nodular center to the edge, the angle of incidence gradually increases from zero to the maximum angle $\theta$, as shown in Figure 1. The equation for determining the angle $\theta$ of $D=\operatorname{sqrt}(4 d t)$ aspect ratio nodules was deduced to be

$$
\theta=\arcsin \left(\frac{2 \sqrt{d t}}{d+t}\right)
$$

An interesting finding is that the incident angular range (IAR) of the $D=\operatorname{sqrt}(4 d t)$ aspect ratio nodules is much larger than that of the $D=\operatorname{sqrt}(8 d t)$ aspect ratio nodules ${ }^{14}$ for the same seed diameter; a comparison is given in Table 1. Figure 2 shows that the angular reflection bandwidth (ARB) of the $\mathrm{HfO}_{2} / \mathrm{SiO}_{2} \mathrm{HR}$ coatings is limited and is approximately $\pm 56^{\circ}$ and $\pm 44^{\circ}$ for S-polarization and P-polarization, respectively. When the IAR of the nodules is larger than the ARB of the $\mathrm{HfO}_{2} / \mathrm{SiO}_{2} \mathrm{HR}$ coatings, the incident laser beam will penetrate the multilayer stack through the nodular edge. Because the two nodular geometries have different IARs, the portion of the laser beam that can penetrate through the multilayer stack will be different, likely resulting in different $|E|^{2}$ enhancement.

The link between $|E|^{2}$ distributions and the damage morphologies of the artificial nodules

Although the nodules prepared via EBE exhibited a $D=\operatorname{sqrt}(4 d t)$ geometry, $|E|^{2}$ simulations for $D=\operatorname{sqrt}(8 d t)$ nodular geometry were also

Table 1 IAR of nodules $\left({ }^{\circ}\right)$

\begin{tabular}{llllll}
\hline Seeds $(\mu \mathrm{m})$ & 0.3 & 0.6 & 0.9 & 1.45 & 1.9 \\
$D=$ sqrt(4dt) & 21 & 30 & 49 & 60 & 67 \\
$D=$ sqrt(8dt) & 30 & 41 & 40 & 45 & 50 \\
\hline
\end{tabular}




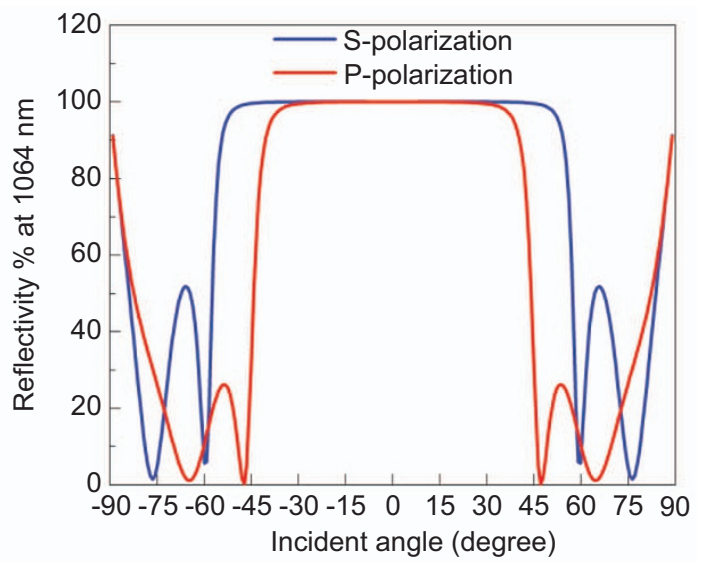

Figure $2 \mathrm{ARB}$ of the $\mathrm{HfO}_{2} / \mathrm{SiO}_{2} \mathrm{HR}$ coatings. The reflection bandwidth is approximately $\pm 56^{\circ}$ for S-polarization and approximately $\pm 44^{\circ}$ for P-polarization. ARB, angular reflection bandwidth; $\mathrm{HR}$, high-reflection.

investigated here for two reasons. First, the $D=\operatorname{sqrt}(8 d t)$ nodular geometry can be used as a benchmark for the correctness of our FDTD code by comparing our $|E|^{2}$ simulation results with those in the literature. Second, comparative $|E|^{2}$ simulations of two nodular geometries can better illustrate the effect of geometry on $|E|^{2}$ distributions in nodules.

Figure 3 shows the S-polarized $|E|^{2}$ distributions for the two nodular geometries initiating from $0.3,0.6,0.9,1.45$ and $1.9 \mu \mathrm{m}$ silica microspheres. The differences between the $|E|^{2}$ distributions for the two nodular geometries are dramatic. For the $D=\operatorname{sqrt}(8 d t)$ nodular geometry, $|E|^{2}$ distributions calculated by our FDTD code were quite similar to the results given by Stolz et al. ${ }^{14}$ For all nodules, the maximum $|E|^{2}$ was located at the upper central axis of the nodules; also, the $|E|^{2}$ distribution gradually increased with increasing seed diameter. Moreover, for perfect HR coatings, the $|E|^{2}$ distribution obtained using our FDTD code also showed excellent agreement with that calculated analytically using Optilayer software. ${ }^{23}$ These results suggest that our FDTD code should be valid for the determination of $|E|^{2}$ distributions in the vicinity of nodules. Compared to the $D=\operatorname{sqrt}(8 d t)$ nodular geometry, the $D=\operatorname{sqrt}(4 d t)$ nodular geometry resulted in very different $|E|^{2}$ distributions. The positions of the $|E|^{2}$ maxima were much deeper and even occurred within the seeds for the larger nodules. Moreover, the $D=\operatorname{sqrt}(4 d t)$ nodular geometry also led to much larger $|E|^{2}$ enhancement than the $D=\operatorname{sqrt}(8 d t)$ nodular geometry. The maximum $|E|^{2}$ values normalized to the incident field intensity for both geometries are given in Table 2.

The observed differences in the $|E|^{2}$ distributions can be qualitatively explained as follows. The IAR of the $D=\operatorname{sqrt}(8 d t)$ aspect ratio nodules is either smaller or slightly larger than the P-polarized ARB of the $\mathrm{HfO}_{2} / \mathrm{SiO}_{2} \mathrm{HR}$ coatings. Because only a small portion of the laser beam can penetrate into the depths of the nodules, the hotspots are located in the upper regions of nodules. For larger $D=\operatorname{sqrt}(4 d t)$ aspect ratio nodules, the IAR is larger than the P-polarized $\mathrm{ARB}$ and even larger than the $\mathrm{S}$-polarized $\mathrm{ARB}$ of the $\mathrm{HfO}_{2} / \mathrm{SiO}_{2} \mathrm{HR}$ coatings; thus, a large portion of the laser beam can penetrate through the multilayer stacks. When the transmitted light arrives at the central axis coherently and in phase, it can create an electric-field enhancement. The highest $|E|^{2}$ can be 18 times higher than the normalized incident $|E|^{2}$. The $D=\operatorname{sqr}(4 d t)$ aspect ratio nodules function like microlenses; they induce much stronger light intensification than the $D=\operatorname{sqrt}(8 d t)$ aspect ratio nodules. Because we are more interested in the $|E|^{2}$ peaks located at the central axis of the nodules, the S-polarized and P-polarized $|E|^{2}$ distributions are very similar for this perspective. The S-polarized $|E|^{2}$ distributions are provided as an example in Figure 3, and the P-polarized $|E|^{2}$ distributions are provided in the supplementary data for reference.

The comparisons above clearly indicate that nodular geometry has a significant influence on the $|E|^{2}$ enhancement and distributions. We believe that the $|E|^{2}$ distributions can significantly affect the damage behaviors of nodules and that the damage morphologies of nodules should reflect the characteristics of the $|E|^{2}$ distributions. To the best of our knowledge, this hypothesis has never been directly proven from experimental results. In this study, we try to find a link between the $|E|^{2}$ distributions and the damage morphologies of nodules and demonstrate exactly how $|E|^{2}$ distributions affect the thermomechanical damage of nodules.

Table 3 provides the ejection fluence $(\mathrm{EF})$ of the artificial nodules. Because nodules initiating from the $0.3 \mu \mathrm{m}$ and $0.6 \mu \mathrm{m}$ seeds could not be damaged at the maximum fluence of our laser damage test facility, our interest here was mainly with the vulnerable nodules initiating from bigger seeds. An FIB instrument was used to examine the crosssectional damage morphologies of the ejected nodules. Figure 4 shows the damage morphologies of nodules initiating from 0.9, 1.45 and $1.9 \mu \mathrm{m}$ silica seeds. The damage morphologies in the left-hand column were considered to be the representative ones and are discussed first. Upon careful examination of the damage morphologies of the nodules initiating from the 1.9- and $1.45-\mu \mathrm{m}$ seeds, we reached the conclusion that the silica seeds were not ejected but rather melted. Only when strong light intensification occurs in seeds, as is the case in our simulation results, can such melting-like damage morphologies occur for silica seeds whose absorption is extremely low. Interestingly, for the damage morphology of the nodule initiating from the $0.9-\mu \mathrm{m}$ silica seed, the nodule was not ejected along the most unstable boundaries, i.e., in the vicinity of the seed, but was instead ejected from the upper, more continuous boundaries. Although the micrographs in Figure 4a1 do not exhibit obvious melting-like damage morphology, we still can infer that the initial damage initiated at the middle region of the film stack where the coating materials melted due to intense $|E|^{2}$, in agreement with the simulation results.

A two-step damage process is proposed. A light intensification focal spot in either seeds or films generates a high temperature. When the temperature exceeds the melting point of the materials, the materials are first melted, and then, the melted regions generate considerable pressure and induce high tensile stress at the boundaries of the nodules, thereby ejecting the mechanically unstable multilayer stacks above the melted regions, leaving intact the remaining materials beneath the melted regions. The observed correlations between the $|E|^{2}$ distributions and the damage morphologies of the artificial nodules are believed to be the first direct evidence proving the correctness of the simulated $|E|^{2}$ distributions and demonstrating exactly how the $|E|^{2}$ distributions affect thermomechanical damage of nodules.

Although we attempted to make identical nodules by creating them from monodisperse silica microspheres, the EF and damage morphologies of nodules initiating from the same-sized seeds still exhibited some differences. The images in the right-hand column of Figure 4 show the complexity of nodular damage. These damage morphologies did not show a good relationship to simulated $|E|^{2}$ distributions, and they were thought to be unrepresentative. For example, the $1.9-\mu \mathrm{m}$ seed did not melt when the film stack was ejected along the boundaries, the $1.45-\mu \mathrm{m}$ seed did not show obvious melting-like damage morphology, and 
S-polarized $|E|^{2}$ profiles

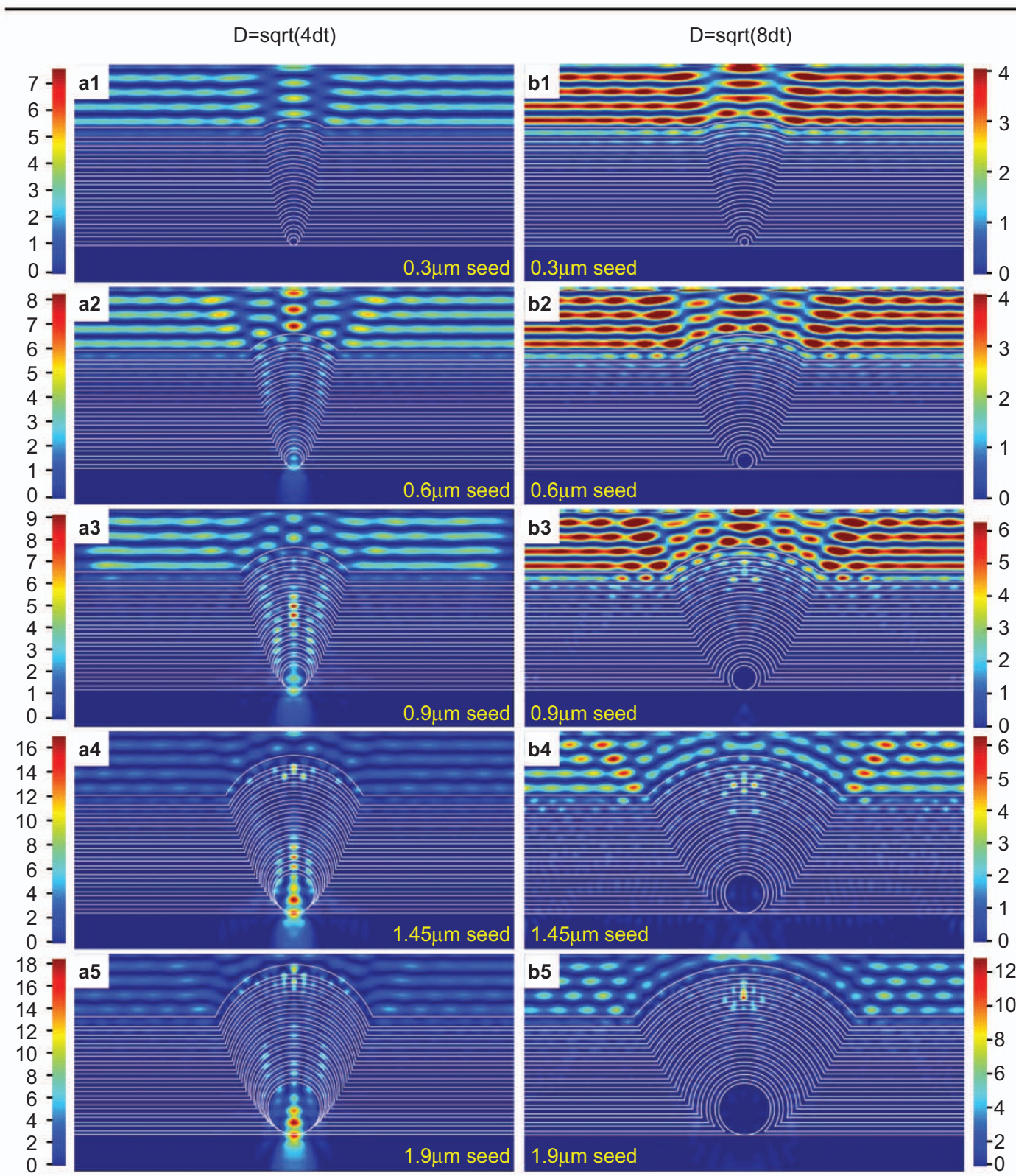

Figure 3 FDTD-simulated S-polarized $|E|^{2}$ distributions at the $y=0 x z$ plane: (a1-a5) for the $D=s q r t(4 d t)$ nodular geometry and (b1-b5) for the $D=s q r t(8 d t)$ nodular geometry. The white lines represent film stacks. To better illustrate the $|E|^{2}$ distributions, the color scale is different for different nodules. FDTD, finitedifference time-domain.

there was no evidence that the initial damage started from the middle of the film stack for the nodule initiating from the 0.9$\mu \mathrm{m}$ seed. We think that the main reason for this complexity is the poor mechanical stability of nodules prepared via EBE. When there is fluctuation in the seed diameter, a slight variation of seed absorption or some other unknown changes, mechanical instability

Table 2 Maximum $|E|^{2}$ in nodules

\begin{tabular}{llllll}
\hline Seeds $(\mu \mathrm{m})$ & 0.3 & 0.6 & 0.9 & 1.45 & 1.9 \\
$D=$ sqrt(4dt) & 4.0 & 4.0 & 5.5 & 16.8 & 17.8 \\
$D=$ sqrt(8dt) & 4.0 & 4.0 & 4.0 & 5.4 & 12 \\
\hline
\end{tabular}

will combine the impact of all fluctuations and result in different damage morphologies, which actually have a negative effect on our judgment of the impact of $|E|^{2}$ distributions on nodular damage.

Two more nodules initiating from $1.9-\mu \mathrm{m}$ seeds were examined via FIB technology. The seeds showed melting-like damage morphologies, and the micrographs are given in the supplementary data. However, the FIB measurement is quite expensive and time-consuming, and it is

Table 3 Ejection fluence of nodules $\left(\mathrm{J} \mathrm{cm}^{-2}, 10 \mathrm{~ns}\right)$

\begin{tabular}{llllll}
\hline Seeds $(\mu \mathrm{m})$ & 0.3 & 0.6 & 0.9 & 1.45 & 1.9 \\
$D=$ sqrt(4dt) & $>170$ & $>170$ & $130 \pm 15$ & $55 \pm 5$ & $35 \pm 5$ \\
\hline
\end{tabular}



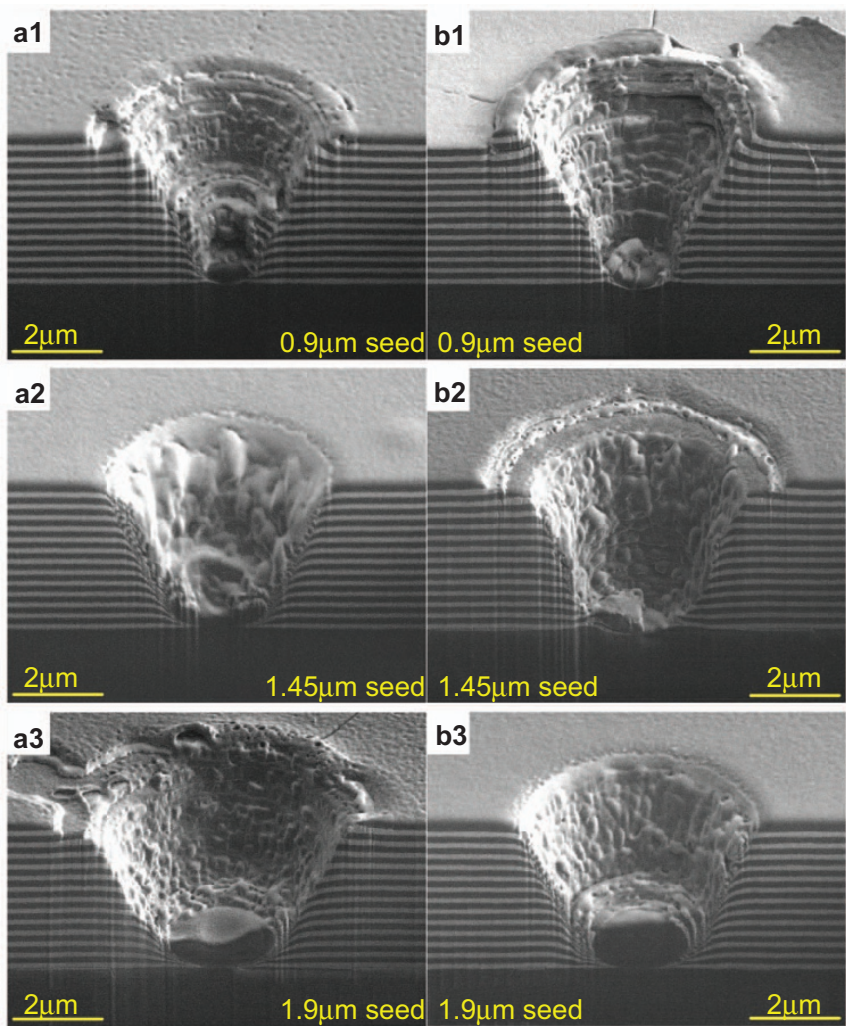

Figure 4 Damage morphologies of nodules revealed by FIB technology. (a1-a3) Damage morphologies that exhibited good agreement with the simulated $|E|^{2}$ distributions. (b1-b3) Damage morphologies that showed some deviation from the simulated $|E|^{2}$ distributions, reflecting the fact that nodular damage is a complicated thermomechanical process. FIB, focused ion beam.

impossible to ascertain the representative damage morphologies by exposing a large number of nodules. Therefore, we prepared another series of nodules to demonstrate the influence of $|E|^{2}$ distributions on the damage behavior of the nodules more efficiently. The basic idea was to make the boundaries more continuous to reduce the influence of mechanical instability. Meanwhile, film absorption was purposely increased to amplify the contribution of the electric field to nodular damage. Ion-assisted deposition (IAD) was used to meet these requirements.

Thermomechanical damage of artificial nodules prepared via IAD To obtain more continuous boundaries and absorbing films, we purposely selected IAD process parameters for high-energy bombardment. ${ }^{18}$ IAD increased the refractive indices of the hafnia and silica films, which reduced the total thickness of the quarter-wave $\mathrm{HfO}_{2} /$ $\mathrm{SiO}_{2}$ HR coating [air: $\mathrm{L}(\mathrm{LH})^{\wedge}{ }^{13}$ glass] to $4.17 \mu \mathrm{m}$. Because the boundaries between the nodules and the surrounding films gradually heal as the film grows, the total thickness of the stack may have some influence on the mechanical stability and the damage behavior of the nodules. Thus, we decided to fix the total film thickness at $4.32 \mu \mathrm{m}$ and to modify the stack structure to be [air: $\mathrm{L}(\mathrm{LH})^{\wedge} 131.3 \mathrm{H}$ glass]. The absorption of the HR coatings prepared via IAD was approximately 40 parts per million at a normal incidence of $1.064 \mu \mathrm{m}$, which is about nine times higher than the absorption of the HR coatings prepared via EBE. This result means that for a given $|E|^{2}$, the absorbed heat or temperature increase in IAD coatings will increase dramatically, which will amplify the impact of $|E|^{2}$ distributions on nodular damage.

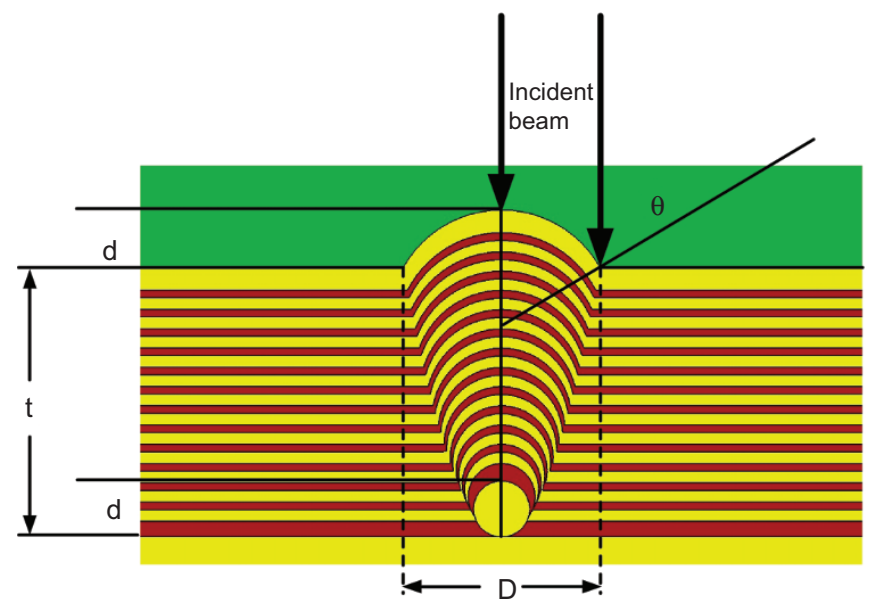

Figure 5 Geometry of a $D=\operatorname{sqrt}(2.5 d t)$ nodule initiating from a $0.9-\mu \mathrm{m}$ silica microsphere. The non-uniformity of the first two layers over the seed is so pronounced that the first two layers are discontinuous at the edge of the seed.

The cross-sectional micrographs of nodules prepared via IAD have been previously examined. ${ }^{18}$ The nodular boundary continuity was actually improved, as expected. A smaller aspect ratio of $D=\operatorname{sqrt}(2.5 d t)$ was found, in accordance with the simulations that found that a higher mobility of deposited atoms would result in smaller nodules. The three conditions introduced above to establish the geometrical model for the $D=\operatorname{sqrt}(4 d t)$ nodules were also adopted here to build a geometrical model for the $D=\operatorname{sqrt}(2.5 d t)$ nodules. Figure 5 shows the simplified cross-sectional geometrical model for a $D=\operatorname{sqrt}(2.5 d t)$ nodule initiating from a $0.9-\mu \mathrm{m}$ seed. For this geometrical model, the non-uniformity of the film thickness over the spherical seeds was even more pronounced than that for the $D=\operatorname{sqr}(4 d t)$ geometrical model.

One particular aspect of the $D=\operatorname{sqrt}(2.5 d t)$ geometrical model is that the intersections between the seeds and the first two deposited layers occur within the seeds; however, this is physically impossible. As a result, the diameters of the first two deposited layers were determined by the seed chord at the depth of the layer thickness where the constraint condition of $D=\operatorname{sqrt}(2.5 \mathrm{dt})$ could not be met. In addition, the IAR of the $D=\operatorname{sqrt}(2.5 d t)$ nodules is also larger than that of the $D=\operatorname{sqrt}(4 d t)$ nodules for the same seed diameter, so a greater portion of the laser beam will penetrate through the multilayer stack along the edge sections of the $D=\operatorname{sqrt}(2.5 d t)$ nodules. Table 4 gives the IAR, maximum $|E|^{2}$ and EF of nodules prepared via IAD. Compared to the values in Tables 1 and 2, we can see that a larger IAR generally induced a stronger $|E|^{2}$ enhancement in the nodules. However, for the case of the $1.45-\mu \mathrm{m}$ seed, the maximum $|E|^{2}$ for the $D=\operatorname{sqrt}(2.5 d t)$ geometry was slightly lower than that for the $D=\operatorname{sqrt}(4 d t)$ geometry. This result cannot be explained solely in terms of IAR, and some unknown factors have some effect here. Compared to the EF in Table 3, the higher absorption of films prepared via IAD significantly decreased the

Table $4 D=\operatorname{sqrt}(2.5 d t)$ nodules

\begin{tabular}{llllll}
\hline Seeds $(\mu \mathrm{m})$ & 0.3 & 0.6 & 0.9 & 1.45 & 1.9 \\
$\operatorname{IAR}\left({ }^{\circ}\right)$ & 29 & 38 & 58 & 70 & 76 \\
Maximum $|E|^{2}$ & 4.5 & 6.5 & 8.5 & 16.2 & 19.5 \\
$\mathrm{EF}\left(\mathrm{J} \mathrm{cm}^{-2}, 10 \mathrm{~ns}\right)$ & $130 \pm 15$ & $85 \pm 10$ & $50 \pm 5$ & $25 \pm 5$ & $15 \pm 5$ \\
\hline
\end{tabular}

Abbreviations: EF, ejection fluence; IAR, incident angular range. 
damage resistance of the nodules, which implies that the joint contribution from $|E|^{2}$ and film absorption determines the EF of the nodules.

The ejection of nodules initiating from $0.3-\mu \mathrm{m}$ seeds always occurred with catastrophic damage, and it was impossible to find or inspect subtle damage morphologies of ejected nodules. Here, we investigated the link between $|E|^{2}$ distributions and damage morphologies of nodules initiating from $0.6,0.9,1.45$ and $1.9 \mu \mathrm{m}$ silica seeds. Figure 6 shows the S-polarized $|E|^{2}$ distributions and the corresponding nodular damage morphologies. The P-polarized $|E|^{2}$ profiles are provided in the supplementary data for reference. The most obvious change of the $|E|^{2}$ distributions for the $D=\operatorname{sqrt}(2.5 d t)$ geometry is that the peak $|E|^{2}$ positions shift upward for some distance compared to the $D=\operatorname{sqrt}(4 d t)$ geometry. Now, most strong $|E|^{2}$ regions are located within the film stack. Because absorption in the film is much higher, the impact of the electric field on nodular damage will be amplified.
The damage morphologies of nodules initiating from 1.9- and $1.45-\mu \mathrm{m}$ seeds have two notable features. The first is that neither the seeds nor deeper layers were melted, but rather were cut apart in the middle. The second is that the film stack was not ejected along existing boundaries, but was destroyed around the central part of the film stack. These features can be perfectly explained from the $|E|^{2}$ distributions, film absorption and mechanical stability. The absorbed heat or temperature increase was determined by the combined effect of $|E|^{2}$ and film absorption. Because the absorption of films prepared via IAD was much higher than that of the seeds, the temperature peak should occur in the film stack according to the simulated $|E|^{2}$ profiles. Moreover, the temperature gradient and stress response should be much more localized to the $|E|^{2}$ distributions due to the high absorption of the films. The film stack melted first, and then a strong tensile stress was generated along the axis of the nodules, which was intense enough to cut open either the
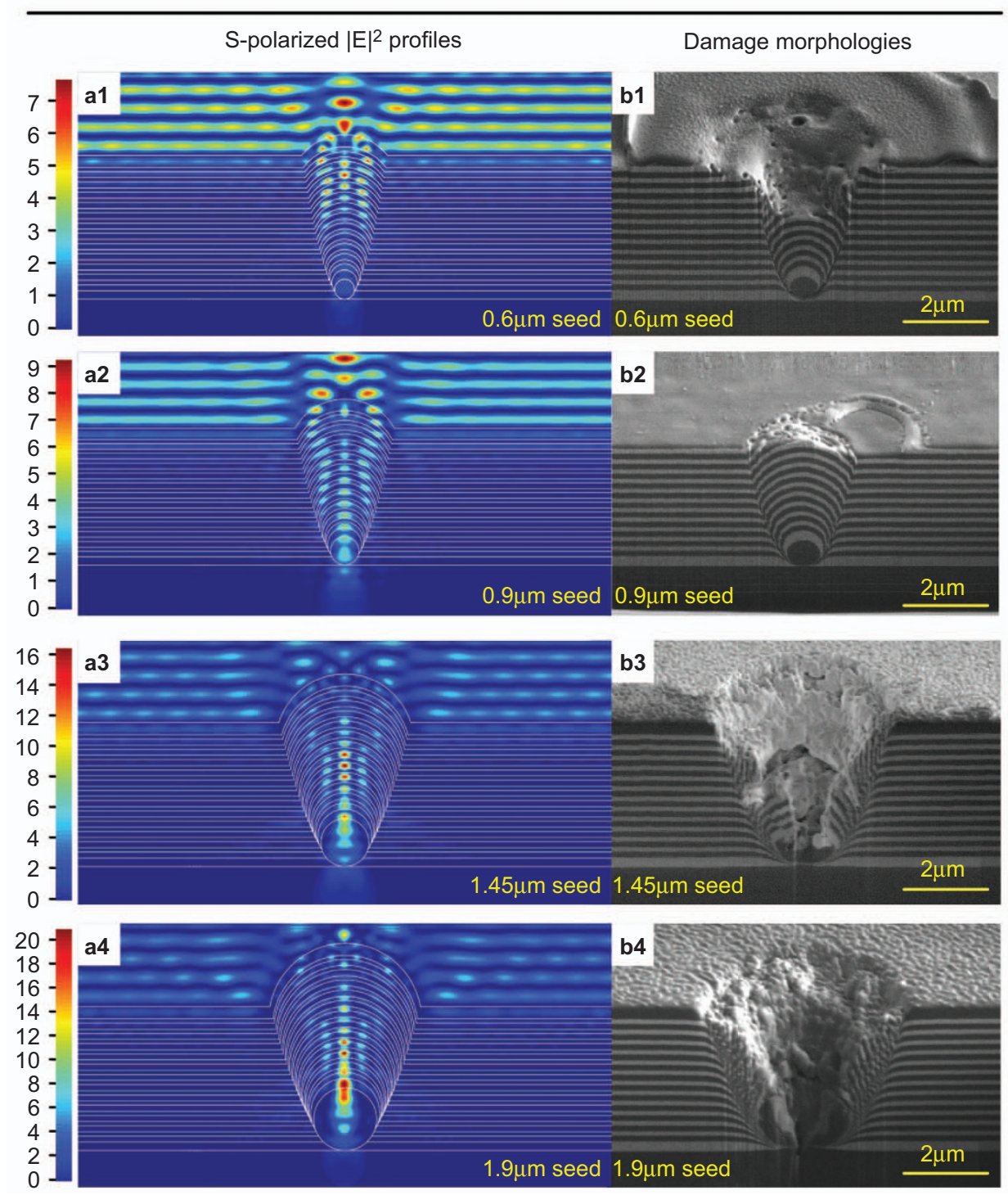

Figure 6 Comparisons between simulated $|E|^{2}$ distributions and the damage morphologies of nodules prepared via IAD. (a1-a4) FDTD-simulated S-polarized $|E|^{2}$ distributions for which white lines represent film stacks and the color scale is different for different nodules. (b1-b4) Damage morphologies that almost exactly reproduce the FDTD-simulated S-polarized $|E|^{2}$ distributions. FDTD, finite-difference time-domain; IAD, ion-assisted deposition. 
deep layers or the seeds. Because boundary continuity was significantly improved during IAD, the coating materials were merely sprayed out near the melting region instead of at the boundaries. It is also interesting to compare Figures $6 \mathrm{~b} 3$ and $6 \mathrm{~b} 4$ with Figure $4 \mathrm{a} 1$. Although the $|E|^{2}$ distributions of these three nodules were similar, the damage morphologies were quite different due to different film absorption and mechanical stability.

Nodules initiating from $0.9-\mu \mathrm{m}$ seeds also showed unique damage morphologies, with only the top several layers being destroyed. This result is in excellent agreement with the simulated $|E|^{2}$ profiles in which the positions of peak $|E|^{2}$ are only located at the top several layers. Because the damaged surface was relatively flat, it was the only type of damage morphology that could be easily verified by scanning electron microscopy without also needing to examine the crosssectional micrographs using FIB. Almost all nodules initiating from the $0.9-\mu \mathrm{m}$ seeds exhibited such damage morphologies, which strongly supported the reliability and validity of the observed link between $|E|^{2}$ distributions and damage morphologies. As for the nodules initiating from the $0.6-\mu \mathrm{m}$ seeds, the strong $|E|^{2}$ positions were located at the upper part of the film stack, so, naturally, the upper part of the film stack melted and was destroyed.

For nodules prepared via IAD, the damage morphologies almost completely reproduced the $|E|^{2}$ distributions, which further proved the correctness of the $|E|^{2}$ simulations and also demonstrated the influence of $|E|^{2}$ distributions on the damage behavior of the nodules irradiated by nanosecond laser pulses. These results also reveal the thermomechanical characteristics of nodular damage and could explain quite well why the EFs of nodules prepared via IAD were much lower than those of nodules prepared via EBE.

\section{CONCLUSIONS}

The impact of $|E|^{2}$ distributions on the thermomechanical damage of nodules has been studied in a comparative manner by both numerical and experimental approaches. A 3D FDTD code was utilized to simulate the $|E|^{2}$ distributions in artificial nodules. The results showed that different nodular geometries exhibited different IARs and resulted in completely different $|E|^{2}$ distributions. The damage morphologies of the two series of artificial nodules prepared via EBE and IAD were explored, and excellent agreement occurred between the damage morphologies and the simulated $|E|^{2}$ distributions. This agreement in results convincingly proved the soundness of our approach to simulate $|E|^{2}$ enhancement and distributions. Neglecting voids along boundaries seemed to have no significant influence on the validity of the $|E|^{2}$ profiles, but the presence of voids might cause variations in the damage morphologies of nodules that were created from similarly sized seeds. More importantly, our results are believed to be the first experimental evidence that demonstrates exactly how $|E|^{2}$ distributions affect the damage behavior of nodules. Our findings provide more information on the damage process of nodules, provide a good foundation for subsequent thermal, stress and mechanical simulations, and also suggest a future direction for increasing the damage resistance of nodules by minimizing $|E|^{2}$ enhancement.

\section{ACKNOWLEDGMENTS}

This work was partly supported by the National Natural Science Foundation of China (Grant Nos. 61235011, 61008030, 61108014, 61205124), the ChenGuang Project of Shanghai Municipal Education Commission (Grant No. 10CG19), the Specialized Research Fund for the Doctoral Program of High Education (Grant No. 20100072120037) and the National 863 Program.

1 Stolz CJ. The National ignition facility: the world's largest optical system. Proc SPIE 2007; 6834: 683402.

2 Besnard D. The megajoule laser program-ignition at hand. Eur Phys J D 2007; 44: 207-213.

3 Yu HW, Jing FG, Wei XF, Zheng WG, Zhang XM et al. Status of prototype of SG-III highpower solid-state laser. Proc SPIE 2009; 7131: 713112.

4 Carr CW, Radousky HB, Demos SG. Wavelength dependence of laser-induced damage: determining the damage initiation mechanisms. Phys Rev Lett 2003; 91: 127402.

5 Jensen L, Mende M, Schrameyer S, Jupé M, Ristau D. Role of two-photon absorption in $\mathrm{Ta}_{2} \mathrm{O}_{5}$ thin films in nanosecond laser-induced damage. Opt Lett 2012; 37: 43294331.

6 Papernov S, Tait A, Bittle W, Schmid AW, Oliver JB et al. Near-ultraviolet absorption and nanosecond-pulse-laser damage in $\mathrm{HfO}_{2}$ monolayers studied by submicrometerresolution photothermal heterodyne imaging and atomic force microscopy. J Appl Phys 2011; 109: 113106.

7 Gallais L, Mangote B, Commandré M, Melninkaitis A, Mirauskas J et al. Transient interference implications on the subpicosecond laser damage of multidielectrics. Appl Phys Lett 2010; 97: 051112.

8 Miller PE, Bude JD, Suratwala TI, Shen N, Laurence TA et al. Fracture-induced subbandgap absorption as a precursor to optical damage on fused silica surfaces. Opt Lett 2010; 35: 2702-2704.

9 DeMange P, Negres RA, Rubenchik AM, Radousky HB, Feit MD et al. Understanding and predicting the damage performance of $\mathrm{KD}_{\mathrm{x}} \mathrm{H}_{2-\mathrm{x}} \mathrm{PO}_{4}$ crystals under simultaneous exposure to 532- and 355-nm pulses. Appl Phys Lett 2006; 89: 181922.

10 Brett MJ, Tait RN, Dew SK, Kamasz S, Labun AH. Nodular defect growth in thin films. J Mater Sci: Mater Electron 1992; 3: 64-70.

11 Tench RJ, Chow R, Kozlowski MR. Characterization of defect geometries in multilayer optical coatings. Proc SPIE 1994; 2114: 415-425.

12 Liao BJ, Smith DJ, Mcintyre B. The formation and development of nodular defects in optical coatings. NBS SP 1987; 746: 305-318.

13 DeFord J F, Kozlowski MR. Modeling of electric-field enhancement at nodular defects in dielectric mirror coatings. Proc SPIE 1993; 1848: 455-472.

14 Stolz CJ, Génin FY, Pistor TV. Electric-field enhancement by nodular defects in multilayer coatings irradiated at normal and $45^{\circ}$ incidence. Proc SPIE 2004; 5273 : 41-49.

15 Stolz CJ, Feit MD, Pistor TV. Laser intensification by spherical inclusions embedded within multilayer coatings. Appl Opt 2006; 45: 1594-1601.

16 Stolz CJ, Feit MD, Pistor TV. Light intensification modeling of coating inclusions irradiated at 351 and $1053 \mathrm{~nm}$. App/ Opt 2008; 47: C162.

17 Shan YG, He HB, Wei CY, Li SH, Zhou M et al. Geometrical characteristics and damage morphology of nodules grown from artificial seeds in multilayer coating. Appl Opt 2010; 49: 4290-4295.

18 Cheng XB, Ding T, He WY, Zhang JL, Jiao HF et al. Using engineered nodules to study laser-induced damage in optical thin films with nanosecond pulses. Proc SPIE 2011; 8190: 819002

19 Sawicki RH, Shang CC, Swatloski TL. Failure characterization of nodular defects in multi-layer dielectric coatings. Proc SPIE 1995; 2428: 333-342.

20 Poulingue M, Ignat M, Dijon J. The effects of particle pollution on the mechanical behaviour of multilayered systems. Thin Solid Films 1999; 348: 215-221.

21 Wei ZY, Cao Y, Han J, Wu C, Fan YC et al. Broadband negative refraction in stacked fishnet metamaterial. Appl Phys Lett 2010; 97: 141901.

22 Cheng XB, Shen ZX, Jiao HF, Zhang JL, Ma B et al. Laser damage study of nodules in electron-beam-evaporated $\mathrm{HfO}_{2} / \mathrm{SiO}_{2}$ high reflectors. Appl Opt 2011; 50: C357C363.

23 Calculated Using Optilayer Thin Film software, Ver. 8.18. http://www.optilayer.com/

(c) This work is licensed under a Creative Commons Attribution-NonCommercial-Share Alike 3.0 Unported License. To view a copy of this license, visit http://creativecommons. org/licenses/by-nc-sa/3.0 